\title{
Time to Line Crossing for Lane Departure Avoidance: a Theoretical Study and an Experimental Setting
}

\author{
Saïd Mammar, Member, IEEE, Sébastien Glaser and Mariana Netto
}

\begin{abstract}
The main goal of this paper is to develop a distance to line crossing (DLC) based computation of time to line crossing (TLC). Different computation methods with increasing complexity are provided. A discussion develops the influence of assumptions generally assumed for approximation. A sensitivity analysis with respect to the vehicle parameters and positioning is performed. For TLC computation, both straight and curved vehicle paths are considered. The road curvature being another important variable considered in the proposed computations, an observer for its estimation is then proposed. An evaluation over a digitalized test track is first performed. Real data is then collected through an experiment carried out in test tracks with our equipped prototype vehicle. Based on these real data, the TLC is then computed with the theoretical proposed methods. Obtained results outlined the necessity to take into consideration vehicle dynamics in order to use the TLC as a lane departure indicator.
\end{abstract}

Index Terms - Time-to-line-crossing, Lane departure avoidance, Driver assistance, Observer.

\section{NOMENCLATURE}

$\begin{array}{ll}\text { CG } & \text { Vehicle center of gravity } \\ m & \text { Vehicle mass (1470 kg) } \\ l_{f} & \text { Distance from CG to front axle }(1.00 \mathrm{~m}) \\ l_{r} & \text { Distance from CG to rear axle }(1.46 \mathrm{~m}) \\ l_{v} & \text { Vehicle base length }\left(l_{f}+l_{r}=2.46 \mathrm{~m}\right) \\ a & \text { Track width }(1.40 \mathrm{~m}) \\ c_{f} & \text { Front cornering stiffness }(41.6 \mathrm{kN} / \mathrm{rad}) \\ c_{r} & \text { Rear cornering stiffness }(47.13 \mathrm{kN} / \mathrm{rad}) \\ \beta & \text { Vehicle sideslip angle (rad) } \\ r & \text { Yaw rate (rad/s) } \\ \delta_{f} & \text { Steering angle (rad) } \\ y_{G} & \text { Distance from lane centerline to CG (m) } \\ y_{G l},\left(y_{G r}\right) & \text { Distance from left (right) boundary of } \\ & \text { the lane to vehicle CG (m) } \\ y_{l l},\left(y_{l r}\right) & \text { Distance from left (right) boundary of } \\ y_{r l},\left(y_{r r}\right) & \text { the lane to front left tire (m) } \\ \psi & \text { Distance from left (right) boundary of } \\ \psi_{L} & \text { the lane to front right tire (m) } \\ \psi_{L}=\psi_{f}+\delta_{f} & \text { Vehicle relative yaw angle (rad) } \\ & \text { Front tire relative yaw angle (rad) }\end{array}$

This work was supported by ARCOS2004 PREDIT project and the European integrated project PReVENT.

S. Mammar is with Université d'Évry val d'Essonne, France. IBISC: Informatique, Biologie Intégrative et Systèmes Complexes - FRE CNRS 2873, 40 rue du Pelvoux CE1455, 91020, Evry, Cedex, France, (e-mail: said.mammar@iup.univ-evry.fr).

M. Netto and S. Glaser are with INRETS/LCPC - LIVIC Laboratoire sur les Interactions Véhicule-Infrastructure-Conducteur. 14, route de la Minière, Bât 824, 78000, Versailles, France, e-mail: ((netto, glaser)@ lcpc.fr).

$R_{r}$
$R_{v}$
$R_{v l},\left(R_{v r}\right)$
$R_{v_{\mathrm{lim}_{l}}}$
$R_{v_{\mathrm{lim}_{r}}}$
$D_{L C}$
$t_{L C}$
$L$
$v_{l}$
$\gamma_{l}$

Road radius of curvature (m)

Vehicle path radius of curvature (m)

Path radius of the front left (right) tire (m)

Maximal vehicle path radius for left

boundary line crossing avoidance (m)

Maximal vehicle path radius for right

boundary line crossing avoidance $(\mathrm{m})$

Distance to line crossing (m)

Time to line crossing ( $\mathrm{sec})$

Lane width $(3.5 \mathrm{~m})$

lateral speed $(\mathrm{m} / \mathrm{s})$

lateral acceleration $\left(\mathrm{m} / \mathrm{s}^{2}\right)$

\section{INTRODUCTION}

Time to line crossing (TLC) is defined as the time duration available for the driver before any lane boundary crossing. Several research studies outlined the importance of this indicator for both driver performance evaluation and lane departure characterization [5], [9]. Among usual observations concerning TLC time evolution, are small TLC values periods prior to lane departure. This happens especially in case of driver drowsiness which generally leads to slow rate TLC decreases with possible presence of one or several TLC local minima corresponding to driver corrections. On the contrary, in the case of vehicle loss control the decreasing of TLC towards zero is generally faster [5], [13]. Unfortunately, real-time computation of TLC is not easy due to several limitations concerning availability of vehicle state variables, vehicle trajectory prediction and lane geometry [16],[17]. Computation time is also a limiting factor. Thus approximate formulas are used, and the usual one is the ratio of lateral distance to lateral speed. This approximation has been successfully used in several works for lane departure systems evaluation [18]. While lateral speed can be not easy to observe, this formula is also not valid when lateral speed varies [22]. A possible way to obtain TLC, which is developed in this paper, is first to compute the distance to line crossing (DLC) along the vehicle path and then to divide it by the vehicle forward speed [13]. This approach, on one hand, presents the advantage not to use the lateral speed which has to be estimated, but on the other hand requires a long preview of the vehicle path and road geometry. DLC based computation method has been used in [21] assuming straight road geometry. However, vehicle path and road profile are both rarely straight and are a succession of curves to the right and to the left. The purpose of this paper is to cover all aspects of TLC including human factors, theoretical as well as real-data computation, highlighting at the same time parameters sensitivity. First, in section II TLC is positioned among other measures also used to study driver performance. 
In section III, some useful geometric formula of TLC are reviewed, first assuming the vehicle in stationary conditions without slipping, and after by taking into account the understeering characteristic of the vehicle through a dynamic model in section IV. Both straight and curved sections are considered and several approximations are provided which can be easily computed in real-time. Finally, a linear dynamic model is used to predict future vehicle positions. An unknown input observer scheme is also proposed in this section in order to estimate the vehicle state and the road curvature which are needed in the prediction phase.

In section $\mathrm{V}$, an evaluation of the TLC is performed on straight and curved road sections. This evaluation is carried out on the basis of the established trigonometric formulas by considering several parameter variations. In section VI, an experimental setting is proposed to collect real data for TLC computation. The evaluation is carried out on a digitalized test track of $3.5 \mathrm{Km}$ long which combines both straight and curved sections. It is shown that the observer is able to estimate the track curvature. We wrap up the paper in section VII with the conclusions.

\section{DRIVER COMMON MEASURES}

In [11], where human-machine interactions aspects are discussed, different sensory information useful for vehicle trajectory control while taking a bend are described. Two anticipation levels concerning these variables can be distinguished. The first level, long-term anticipation, begins as soon as the bend is visible. The second level, short-term anticipation and on-line control, begins just before and during bend taking.

As long-term anticipation visual variables one can cite the visual angle. This variable corresponds to the visual curvature of the road perceived by the driver and is available as soon as the bend is visible.

Concerning short-term anticipation, one can cite for example the optical flow. Changes in the optical flow directly specify interactions between the individual and the environment [4]. Some variables can be derived from the optical flow. A specific point in the optical flow, the tangent point is one of them [10]. It is observed that, during bend taking, the driver tends to fix his view in this point. Another variable derived from the optical flow is the egocentric direction which corresponds to the combination of the extra-retinal information concerning the direction of the gaze (with respect to the axis of the body) and retinal location. Some studies indicate the utilization of the visual egocentric direction in order to guide the displacements (see for example [12]).

Still talking about short-term anticipation, the Time to Line Crossing seems to play an important role as an indicator of steering performance as well as a regulating variable for the driver action (that is, it seems to help the driver's mind to perform the task of driving). With respect to the second case, research about TLC [14], [15] indicates that drivers can compensate for some errors of steering by decreasing their speed in order to maintain the TLC constant. In addition, the TLC seems to be a good indicator of the point at which the driver begins to use a strategy of open-loop visual steering (when he stops to look at the road momentarily to perform another task inside the vehicle) depending on the speed.

In addition to visual information, steering most certainly involves sensory information from various other organs, like vestibular, tactile or proprioceptive organs.

Finally, in [11], it is highlighted the fact that, it is certainly not the same type of information used, depending on the temporality of the task. The question "assistance for what type?" is then raised. The temporal relation of the TLC with the other variables is shown in figure 1 (see [11]). Based on this temporal frame we can distinguish two main classes of assistances : preventive or foresighted driving and short-term decision assistances. This division suggests that the first one would concern mainly informative or warning tasks to help the driver, while the second one could be based on warning as well as on active systems like trajectory correction. The task of driving in this case is mostly performed by the driver and the controller acts for lane departure avoidance. The TLC fits this frame, being fundamental to help to determine in which moment the assistance has to be turned on to avoid the lane departure. In addition, its importance appears also in HMI studies, for drivers trajectory evaluation.

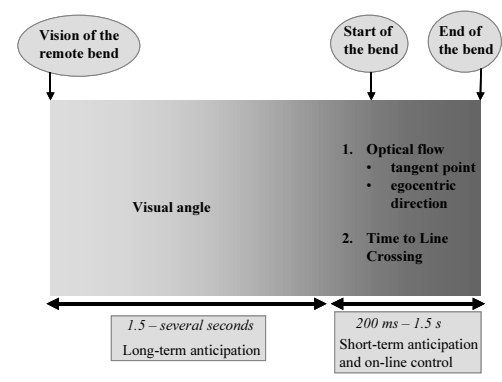

Fig. 1. Temporal representation of visual variables during a bend taking.

\section{GEOMETRIC EQUATIONS}

In this section, expressions of distance to line crossing and time to line crossing are derived using a kinematic model of the car and trigonometric formulas. Straight road and curved road sections are successively examined. In both cases, zero steering angle and constant non-zero steering angle are treated. Approximate solutions are provided in order to clarify the contribution to TLC of non-zero values of steering angle or road curvature.

\section{A. Vehicle positioning}

We derive first the positioning of the vehicle front tires relative to the left and right lane boundaries according to actual vehicle $\mathrm{CG}$ lateral displacement, vehicle geometry and relative yaw angle. Figure 2 summarizes all used conventions.

1) Positioning relative to left lane boundary: Assume that the vehicle $C G$ is at a distance $y_{l}$ from the left line of the lane of a straight road section, with a relative yaw angle $\psi$. Let $y_{l l}$ (resp. $y_{r l}$ ) be the lateral distance of front left (resp. right) 


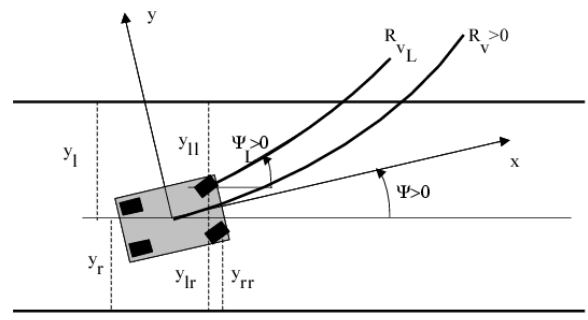

Fig. 2. Common convention used for vehicle positioning.

tire to the line that would be crossed, the following equations hold for each front tire

$$
\left\{\begin{array}{l}
y_{l l}=y_{l}-l_{f} \sin \psi-\frac{a}{2} \cos \psi \\
y_{r l}=y_{l}-l_{f} \sin \psi+\frac{a}{2} \cos \psi
\end{array}\right.
$$

All angles including relative yaw angle and steering angle are counted positive to the left (anti-clockwise). These formula are still valid for curved road section considering that the road radius $R_{r}$ is much larger than $y_{l}$.

2) Positioning relative to right lane boundary: Similar formula are obtained for right lane boundary

$$
\left\{\begin{array}{l}
y_{l r}=y_{r}+l_{f} \sin \psi+\frac{a}{2} \cos \psi \\
y_{r r}=y_{r}+l_{f} \sin \psi-\frac{a}{2} \cos \psi
\end{array}\right.
$$

Notice in addition that $y_{r}=L-y_{l}$, where $L$ is the lane width. Also, when the front wheels steering angle $\delta_{f}$ is non-zero, the front tires relative yaw angle is $\psi_{L}=\psi+\delta_{f}$.

\section{B. Straight road section}

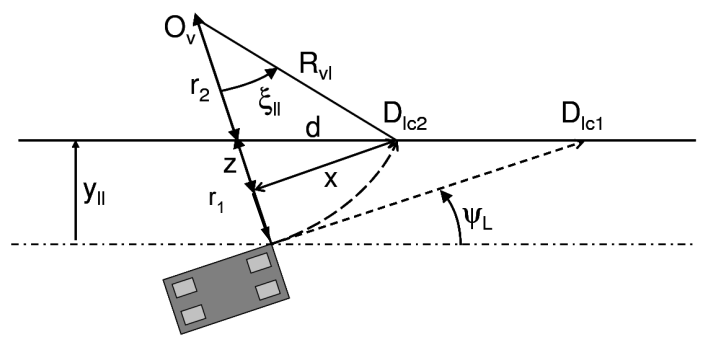

Fig. 3. DLC on straight road section using straight and circular vehicle path.

1) Zero steering angle: Assuming that the steering angle is zero, the vehicle goes straight (figure 3 ). In the case the line crossing occurs on the left side of the lane, the distance to line crossing $D_{L C}$ is simply computed from

$$
D_{L C}=\frac{y_{l l}}{\sin \psi}=\frac{y_{l}-l_{f} \sin \psi-\frac{a}{2} \cos \psi}{\sin \psi}
$$

This formula is valid if $\psi$ is positive. When $\psi$ is negative, one has to use instead the vehicle front right tire distance from the right boundary of the lane $\left(y_{r r}\right)$.
Time to line crossing is obtained by dividing $D_{L C}$ by vehicle speed $v$. For a vehicle leaving to the left side of the lane, it is given by

$$
t_{L C}=\frac{y_{l l}}{v \sin \psi}
$$

Since $v \sin \psi=v_{l}$ is the lateral velocity, we have that $t_{L C}=$ $\frac{y_{l l}}{v_{l}}$ which is the common formula used in the literature [18].

We provide here another formula for TLC assuming that the lateral displacement is subject to a small constant acceleration $\gamma_{l}$ which appears during driver corrective maneuvers

$$
t_{L C}=\frac{1}{\gamma_{l}}\left(-v \sin \psi+\sqrt{\left(v^{2} \sin ^{2} \psi+2 \gamma_{l} y_{l l}\right)}\right)
$$

The series expansion around $\gamma_{l}=0$ gives

$$
t_{L C}=\frac{y_{l l}}{v \sin \psi}\left(1-\frac{1}{2} \frac{y_{l l}}{v^{2} \sin ^{2} \psi} \gamma_{l}\right)
$$

that reduces to the former expression when $\gamma_{l}=0$.

Let us consider now a vehicle circular path which corresponds to constant non-zero steering angle.

2) Constant steering angle: The vehicle steering angle is now constant and positive non-zero and is equal to $\delta_{f 0}$. In steady state, the front left tire path is a circle arc of radius $R_{v_{l}}$ such that

$$
R_{v_{l}}=\frac{l_{v}}{\tan \delta_{f 0}}-\frac{a}{2}=\frac{v}{\dot{\psi}}-\frac{a}{2}
$$

where $l_{v}$ is the vehicle base length and $\dot{\psi}$ is the yaw rate. Notice that, as indicated above, the front left tire presents a relative yaw angle of $\left(\psi_{L}=\psi+\delta_{f_{0}}\right)$

The distance to line crossing is obtained from the following process

1) Compute $r_{1}=\frac{y_{l l}}{\cos \psi_{L}}$ and $r_{2}=R_{v_{l}}-r_{1}$ (figure 3)

2) Compute the distance $d$ from

$$
d=r_{2} \sin \psi_{L}+\sqrt{R_{v_{l}}^{2}-r_{2}^{2} \cos ^{2} \psi_{L}}
$$

3) Compute the angle $\xi_{l l}$ as

$$
\xi_{l l}=\cos ^{-1}\left(\frac{r_{2}^{2}+R_{v_{l}}^{2}-d^{2}}{2 R_{v_{l}} r_{2}}\right)
$$

Finally the distance to line crossing is obtained as

$$
D_{L C}=\xi_{l l} R_{v_{l}}
$$

When $\psi_{L}=0$, the previous equations reduce to $r_{1}=y_{l l}, r_{2}=$ $R_{v_{l}}-y_{l l}, d=\sqrt{R_{v_{l}}^{2}-r_{2}^{2}}$ and $\xi_{l l}=\cos ^{-1}\left(\frac{r_{2}}{R_{v_{l}}}\right)$

The previous equations are established using distance formulas. It is however possible to obtain more simple equations. According to figure $3, \sin \psi_{L}=\frac{z}{d}, \cos \psi_{L}=\frac{x}{d}$, $\cos \xi_{l l}=\frac{r_{2}+z}{R_{v_{l}}}$ and $\sin \xi_{l l}=\frac{x}{R_{v_{l}}}$. Combining the four previous equations leads to

$$
\xi_{l l}=\cos ^{-1}\left(\cos \psi_{L}-\frac{y_{l l}}{R_{v_{l}}}\right)-\psi_{L}
$$

If $\psi_{L}$ and $\delta_{f}$ are both negative, the lane departure should occur on the right side of the lane. The formulas in this case 
are similar, but lateral displacement has to be taken relative to the right lane at the point of contact of the front right wheel.

Suppose now that $\psi_{L}$ and $\delta_{f}$ are of opposite sign, as when the driver performs a recovering maneuver. In such a case, the vehicle may first leave the line on one side and then come back to the lane and finally cross the line on the other side. However, there exists a relation between $R_{v_{l}}, y_{l l}, \psi_{L}$ and the lane width $L$ which determines if the first lane departure will occur on one side or on the other. When $\psi_{L}<0$ and $\delta_{f}$ $>0$, the vehicle path radius has to be under a limit value $R_{v_{\lim _{r}}}>0$ so that the lane departure occurs on the left side. Similarly when $\psi_{L}>0$ and $\delta_{f}<0$, the vehicle path radius has to be also under a limit value $R_{v_{\lim _{l}}}<0$ so that the lane departure still occurs on the left side.

Table I summarizes the cases of TLC calculation when lane departure is expected on the left border.

TABLE I

SUMMARY OF TLC FOR LEFT LINE ON STRAIGHT ROAD SECTION

\begin{tabular}{ccccc}
\hline & $\delta_{f}=0$, & $\delta_{f}>0$, & $\delta_{f}>0$, & $\delta_{f}<0$, \\
$\psi>0$ & $\psi_{L}>0$ & \\
$\psi_{L}<0$ & $\psi_{L}>0$ \\
Case & & & & \\
\hline$R_{v_{l}}$ & $\infty$ & $\frac{l_{v}}{\tan \delta_{f 0}}-\frac{a}{2}$ & $<R_{v_{\text {lim }}}$ & $<R_{v_{\text {lim }}}$ \\
& & $\frac{R_{v_{l}}}{v} \xi_{l l}$ & $\frac{R_{v_{l}}}{v} \xi_{l l}$ & $-\frac{R_{v_{l}}}{v} \xi_{l r}$ \\
\hline TLC & $\frac{y_{l l}}{v \sin \psi}$ & & &
\end{tabular}

where $R_{v_{\text {lim }_{l}}}=\frac{-y_{l l}+a \cos \psi}{1-\cos \psi_{L}}<0, R_{v_{\lim _{r}}}=$ $\frac{L-\left(y_{l l}+a \cos \psi\right)}{1-\cos \psi_{L}}$ and $\xi_{l r}=\cos ^{-1}\left(\cos \psi_{L}-\frac{y_{l l}}{R_{v_{l}}}\right)+\psi_{L}$.

A similar table can be drawn up for the lane departure expected on the right side.

\section{Constant radius curved road section}

1) Zero steering angle: Suppose now that the vehicle is on a curved road section of radius $R_{r}$, the steering is zero such that the vehicle continues its trajectory in the direction of its longitudinal axis.

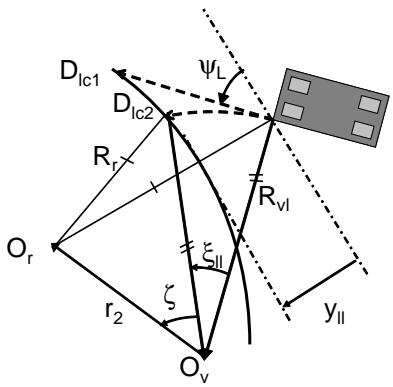

Fig. 4. Vehicle on curved road section with zero and non zero steering angle.

Assuming that the lane departure occurs on the left side of the lane (Figure 4), the computation of $D_{L C}$ is straightforward. One may obtain

$$
D_{L C}=\left(R_{r}+y_{l l}\right) \sin \psi-\sqrt{R_{r}^{2}-\left(R_{r}+y_{l l}\right)^{2} \cos ^{2} \psi}
$$

This solution exists if and only if $\cos \psi \leq \frac{R_{r}}{R_{r}+y_{11}}$. Otherwise, the lane departure occurs on the right side of the lane and $D_{L C}$ is given in this case by

$$
\begin{aligned}
& D_{L C}=\left(R_{r}+y_{l l}+a \cos \psi\right) \sin \psi+ \\
& \sqrt{\left(R_{r}+L\right)^{2}-\left(R_{r}+y_{l l}+a \cos \psi\right)^{2} \cos ^{2} \psi}
\end{aligned}
$$

It is important to notice that on straight road section, when $\delta_{f}=0$, the sign of $\psi$ determines the side of the lane where the lane departure occurs. On curved road section, right side lane departure may occur even if $\psi$ is positive.

Useful approximate formulas that highlight the effect of the road radius of curvature on the distance to line crossing, when the radius of curvature goes to infinity can be derived

- Lane departure on the left side assuming $\psi>0$

$$
D_{L C} \approx \frac{y_{l l}}{\sin \psi}+\frac{1}{2}\left(\frac{\cot ^{2} \psi}{\sin \psi}\right) y_{l l}^{2} \frac{1}{R_{r}}
$$

- Lane departure on the right side assuming $\psi \leq 0$

$$
D_{L C} \approx \frac{y_{r r}}{\sin \psi}-\frac{1}{2}\left(\frac{\cot ^{2} \psi}{\sin \psi}\right) y_{r r}^{2} \frac{1}{R_{r}}
$$

Suppose that $\psi=0$, then the lane departure will occur on the right side of the lane if the road goes to the left. Distance to line crossing is $D_{L C}=\sqrt{\left(L-y_{l l}\right)\left(y_{l l}+L+2 R_{r}\right)}$ and time to line crossing is $t_{L C}=\frac{\sqrt{\left(L-y_{l l}\right)\left(y_{l l}+L+2 R_{r}\right)}}{v}$. On the other hand, at the initial time, the vehicle lateral acceleration is $\gamma_{l}=\frac{v^{2}}{R_{r}+y_{l l}}=\frac{v^{2}}{R_{r}} \frac{1}{1+\frac{y_{l l}}{R_{r}}} \approx \frac{v^{2}}{R_{r}}$. This leads to the equality

$$
t_{L C}=\frac{\sqrt{R_{r}}}{v} \sqrt{\left(L-y_{l l}\right)\left(2+\frac{y_{l l}+L}{R_{r}}\right)}
$$

or assuming $R_{r}>>\left(y_{l l}+L\right)$

$$
t_{L C} \approx \sqrt{\frac{2\left(L-y_{l l}\right)}{\gamma_{l}}}
$$

This formula is similar to that for time to collision $t_{c}$ with an obstacle located at a distance $d$ when the vehicle has a constant braking deceleration of $\gamma_{b}: t_{c}=\sqrt{\frac{2 d}{\gamma_{b}}}$.

Tables II and III summarize all formulas for lane departure on left and right sides. All cases of positive and negative road radius of curvature and vehicle yaw angle errors are covered.

TABLE II

SUMMARY OF TLC FOR LEFT LINE ON CURVED ROAD SECTION ASSUMING STRAIGHT VEHICLE PATH

\begin{tabular}{cccc}
\hline & $R_{r}>0$, & $R_{r}>0$, & $R_{r}<0$, \\
& $\psi_{L}>\psi_{t l}$ & $\psi_{L}=\psi_{t l}$ & $\psi_{L} \geq \psi_{t r}$ \\
& & & \\
& & &
\end{tabular}

In these tables, $D_{1}=c_{1} \sin \psi-\sqrt{R_{r}^{2}-c_{1}^{2} \cos ^{2} \psi}, D_{2}=$ $c_{2} \sin \psi+\sqrt{R_{r}^{2}-c_{2}^{2} \cos ^{2} \psi}, D_{3}=c_{2} \sin \psi-\sqrt{c_{3}^{2}-c_{2}^{2} \cos ^{2} \psi}$ and $D_{4}=c_{4} \sin \psi+\sqrt{c_{3}^{2}-c_{4}^{2} \cos ^{2} \psi} \cdot c_{1}=R_{r}+y_{l l}$, 
TABLE III

SUMMARY OF TLC FOR RIGHT LINE ON CURVED ROAD SECTION ASSUMING STRAIGHT VEHICLE PATH

$\left.\begin{array}{cccc}\hline & R_{r}<0, & R_{r}<0, & R_{r}>0, \\ \psi_{L}<\psi_{t r} & \psi_{L}=\psi_{t r} & \psi_{L} \leq \psi_{t l} \\ \text { Case } & \left(\begin{array}{l}\mid \\ \text { TLC }\end{array}\right. & \frac{\sqrt{-2 y_{r r c_{2}}}}{v} & \end{array}\right)$

$c_{2}=R_{r}+L+y_{r r}, c_{3}=R_{r}+L$ and $c_{4}=R_{r}+y_{r l}$. The angles $\psi_{t r}$ and $\psi_{t l}$ are computed as $\psi_{t r}=-\cos ^{-1}\left(\frac{R_{r}+L}{R_{r}+L-y_{r r}}\right)$ and $\psi_{t l}=\cos ^{-1}\left(\frac{R_{r}}{R_{r}+y_{l l}}\right)$.

2) Constant steering angle: According to figure 4, the arc length is given by $D_{L C}=R_{v_{l}} \xi_{l l}$. Computation of $\xi_{l l}$ is however different in this case. It is achieved by the following process (see figure 4)

- The distance $r_{2}$ between the center point of the road curve and the center point of the vehicle path is given by

$$
r_{2}=\sqrt{\left(R_{r}+y_{l l}\right)^{2}+R_{v_{l}}^{2}-2\left(R_{r}+y_{l l}\right) R_{v_{l}} \cos \psi}
$$

- The angle $\zeta$ is then computed as

$$
\zeta=\cos ^{-1}\left(\frac{R_{v_{l}}^{2}+r_{2}^{2}-R_{r}^{2}}{2 r_{2} R_{v_{l}}}\right)
$$

- Finally, the angle $\xi_{l l}$ is given by

$$
\xi_{l l}=-\zeta+\cos ^{-1}\left(\frac{r_{2}^{2}+R_{v_{l}}^{2}-\left(R_{r}+y_{l l}\right)^{2}}{2 r_{2} R_{v_{l}}}\right)
$$

In the previous computation, it has been assumed that both $\psi_{L}$ and $\delta_{f}$ are positive and that the lane departure occurs on the left side. However this lane departure may not occur on left side even in this case. The limit situation is when the path of the vehicle is tangent to the left border of the lane (figure $5)$. In such a case, the angle $\zeta$ is equal to zero and the two circle centers $O_{v}$ and $O_{r}$ are aligned and the distance between them is $r_{2}=R_{v_{l}}-R_{r} \geq 0$. The corresponding limit value $R_{v_{\lim _{l}}}$ of $R_{v_{l}}$ is given by

$$
R_{v_{\lim _{l}}}=\frac{1}{2} \frac{\left(2+\frac{y_{l l}}{R_{r}}\right) y_{l l}}{-1+\left(1+\frac{y_{l l}}{R_{r}}\right) \cos \psi_{L}}
$$

Note that this is only possible for $\psi_{L}<\cos ^{-1}\left(\frac{R_{r}}{R_{r}+y_{l l}}\right)$. In this case the steering angle has to be less than $\tan ^{-1}\left(\frac{l_{v}}{R_{v_{1 i m_{l}}}}\right)$, in order to avoid a lane departure on the left.

Assume now that $\psi_{L}$ and $\delta_{f}$ are of opposite signs. Without loss of generality, we consider the case $\psi_{L}<0$ and $\delta_{f}>0$, and we determine the condition to avoid lane departure on the right side. Limit value $R_{v_{\text {lim }_{r}}}$ of $R_{v}$ is

$$
R_{v_{\lim _{r}}}=\frac{1}{2} \frac{L\left(2+\frac{L}{R_{r}}\right)-y_{r l}\left(2+\frac{y_{r l}}{R_{r}}\right)}{\left(1+\frac{L}{R_{r}}\right)-\left(1+\frac{y_{r l}}{R_{r}}\right) \cos \psi_{L}}
$$

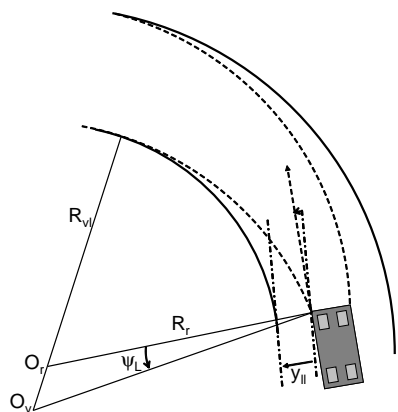

Fig. 5. Limit trajectory for curved road section.

When the vehicle path radius verifies $R_{v_{\lim _{l}}}<R_{v}<$ $R_{v_{\lim _{r}}}$, TLC is infinite since the vehicle trajectory always remains within lane boundaries.

Table IV summarizes the cases of TLC calculation when lane departure is expected on the left border. The road curvature is assumed positive which means that the bend is also oriented to the left.

TABLE IV

SUMMARY OF TLC FOR LEFT LINE ON CURVED ROAD SECTION

\begin{tabular}{cccc}
\hline Case & $\begin{array}{c}\delta_{f}=0, \\
\cos \psi_{L}<\frac{R_{r}}{R_{r}+y_{l l}}\end{array}$ & $\begin{array}{c}\delta_{f}=\delta_{f 0}>0, \\
\cos \psi_{L}<\frac{R_{r}}{R_{r}+y_{l l}}\end{array}$ & $\begin{array}{c}\delta_{f}=\delta_{f 0}>0, \\
\cos \psi_{L}>\frac{R_{r}}{R_{r}+y_{l l}}\end{array}$ \\
\hline$\psi_{v_{l}}>0$ & $\infty$ & $\begin{array}{c}R_{v_{l}}=\frac{l_{v}}{\tan \delta_{f 0}}-\frac{a}{2} \\
<R_{v_{1 i m}}\end{array}$ & $\begin{array}{c}R_{v_{l_{1 i m}}}<R_{v} \\
R_{v}<R_{v_{1 i m}}\end{array}$ \\
\hline TLC & $\frac{1}{v}\left(\sqrt{\left.\frac{\left(R_{r}+y_{l l}\right) \sin \psi-}{R_{r}^{2}-\left(R_{r}+y_{l l}\right)^{2} \cos ^{2} \psi}\right)}\right.$ & Use equation $(20)$ & $\infty$ \\
\hline
\end{tabular}

\begin{tabular}{|c|c|c|c|}
\hline Case & $\begin{array}{c}\delta_{f}=0 \\
\psi_{L} \geq 0 \\
\cos \psi_{L}>\frac{R_{r}}{R_{r}+y_{l l}}\end{array}$ & $\begin{array}{c}\delta_{f}=\delta_{f 0}>0 \\
\cos \psi_{L}>\frac{R_{r}}{R_{r}+y_{l l}}\end{array}$ & $\delta_{f}>0$ \\
\hline$R_{v_{r}}$ & 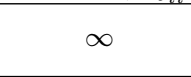 & $\begin{array}{c}R_{v_{r}}=\frac{l_{v}}{\tan \delta_{f 0}}-\frac{a}{2} \\
R_{v_{r}}>R_{v_{\lim _{r}}}\end{array}$ & $\begin{array}{l}R_{v_{r}}>R_{v_{\lim _{l}}} \\
R_{v_{r}}<R_{v_{\lim _{r}}}\end{array}$ \\
\hline TLC & $A$ & similar to $(20)$ & $\infty$ \\
\hline
\end{tabular}

Similarly table $\mathrm{V}$ corresponds to the right side when the bend is still oriented to the left.

TABLE V

SUMMARY OF TLC FOR RIGHT LINE ON CURVED ROAD SECTION

In table $\mathrm{V}, A=\frac{1}{v}\left(\frac{\left(R_{r}+y_{l l}+a \cos \psi_{L}\right) \sin \psi_{L}+}{\sqrt{\left(R_{r}+L\right)^{2}-\left(R_{r}+y_{l l}+a \cos \psi_{L}\right)^{2} \cos ^{2} \psi_{L}}}\right)$. In tables IV and $\mathrm{V}$, only non negative values of $\delta_{f}$ are given, however similar results may be obtained for negative steering angles by providing some substitutions. Analogous results may also be obtained for curved road sections to the right $\left(R_{r}<0\right)$.

\section{Kinematic prediction of vehicle trajectory}

Suppose that the road curvature is made available by the use of an observer for example. Using the lateral positioning and relative yaw angle obtained by the video sensor together with the yaw rate sensor, the steering angle and the speed, it is possible to apply the formula established in section III. The process has to pass through the tables listed above in order to determine in which particular case the vehicle is. It 
is also possible to perform vehicle future positions prediction assuming circular paths. An associated kinematic model which neglects vehicle slip motion can be used. It is given by ( $\dot{x}_{v}=$ $v \cos \theta, \dot{y}_{v}=v \sin \theta, \dot{\theta}=\frac{v}{l_{f}+l_{r}} \tan \delta_{f}$ ), where $\theta$ is the vehicle heading angle, $l_{f}$ and $l_{r}$ are respectively the distance from vehicle CG to front and rear axles, and $\left(x_{v}, y_{v}\right)$ are the vehicle global coordinates. The relative yaw angle dynamics is $\dot{\psi}=$ $\dot{\theta}-\frac{v}{R_{r} \cos \psi}$.

Practically, from CG lateral deviation and relative yaw angle, we determine the front tires path radius and relative yaw angle. As the first concern is to know if TLC is higher than a threshold $S$ or not, one first generates for each of the front tires a circular arc of length $\frac{S i}{N} v(i=1, \ldots, N)$ where $N$ is the number of desired samples. The two lateral deviations are computed for each sample. If, until the $N^{t h}$ sample, they are with in the lane limits, thus TLC is higher than $S$ and the procedure stops. Otherwise, if at time sample $k$, the lateral deviation is found beyond the lane limits, this means that the lane boundary has been crossed between $(k-1)$ and $k$, the minimal TLC is thus $\left(\frac{S(k-1)}{N}\right)$. A refinement is possible in order to obtain a better approximation by using resampling within the time interval $\left[\frac{S(k-1)}{N}, \frac{S k}{N}\right]$.

Notice that the procedure may be accelerated if a straight vehicle path is assumed. An initial line segment of length $\frac{S v}{N}$ can then be directly generated, and lateral deviations are first computed for the last points of the arcs. Thus iteration may be done backward if one is found beyond the boundaries of the lane. Otherwise, we can conclude that TLC is higher than $S$ sec with only two lane positioning.

In the following, the effect of vehicle dynamics on TLC computation is examined. An unknown input proportional multiple integral observer is proposed for vehicle state and road curvature estimation.

\section{PREDICTION USING DYNAMIC MODELS}

\section{A. Vehicle models}

Several models can be used for numerical TLC estimation on various road types. For high longitudinal speeds assuming small angles, a dynamic bicycle linear model can be used. It is formulated in terms of lateral displacement and lateral speed [1]. This model is well fitted for motorway driving conditions. First of all, lateral acceleration written in the road frame is given by

$$
\ddot{y}_{G}=\gamma_{l}-v \frac{1}{R_{r}}=\dot{v}_{l}-v^{2} \rho_{r}
$$

where $\rho_{r}=R_{r}^{-1}$ is the road curvature.

The vehicle path radius $R_{v_{d}}$ of this dynamic model is thus

$$
R_{v_{d}}=\frac{\left(l_{f}+l_{r}\right)}{\delta_{f_{0}}}\left(K v^{2}+1\right)
$$

where $K=\frac{\left(l_{r} c_{r}-l_{f} c_{f}\right) m}{c_{f} c_{r}\left(l_{r}+l_{f}\right)^{2}}$ is a stabilizing factor, $m$ is the vehicle mass, $c_{f}, c_{r}$ are respectively the front and rear tire cornering stiffness. The factor $K$ is positive for an understeer vehicle, negative for an oversteer and zero for a neutral vehicle. Path radius $\left(R_{v_{l}}=\frac{l_{f}+l_{r}}{\tan \delta_{f_{0}}} \approx \frac{l_{f}+l_{r}}{\delta_{f_{0}}}\right)$ obtained with the kinematic model has thus to be corrected.
The temporal relation between the lateral acceleration $\gamma_{l}$, the lateral speed $v_{l}$, the yaw rate $r$ and the steering angle $\delta_{f}$ is

$$
\gamma_{l}=-\frac{\left(c_{f}+c_{r}\right)}{m v} v_{l}+\frac{1}{v} \frac{\left(c_{r} l_{r}-c_{f} l_{f}\right)}{m} r+\frac{c_{f}}{m} \delta_{f}
$$

Under the assumption, $v_{l} \approx v \beta$, which holds for lateral acceleration under $0.3 \mathrm{~g}$, one can notice that the contribution of the yaw rate to lateral acceleration decreases when speed increases in favor to sideslip angle. This means that the vehicle tends to slip rather than to revolve. Thus the computational method of TLC has to be changed.

In addition to the previous established trigonometric formulas, the following formulas can be used for computation of TLC. Only left lane departure cases are provided.

- If lateral speed and lateral acceleration are available for measurement, the first approximate formula which can be used on straight road sections is

$$
t_{L C}=\frac{-v_{l}+\sqrt{v_{l}^{2}+2 \gamma_{l} y_{l l}}}{\gamma_{l}}
$$

This formula is simply derived from the lateral motion of the vehicle assumed to be at a constant lateral acceleration $\gamma_{l}$ with initial lateral speed $v_{l}$.

- On straight road section approximation, taking into account non zero steering angle

$t_{L C}=\frac{l_{v}\left(K v^{2}+1\right) \cos ^{-1}\left(\cos \psi-\frac{y_{l l}}{l_{v}\left(K v^{2}+1\right)} \delta_{f_{0}}\right)-\psi}{v \delta_{f_{0}}}$

This equation is simply derived from the previously trigonometric equation (10) obtained for non-zero steering angle on straight road section, where the vehicle path is replaced in this case by the dynamic one (see table III).

- On curved section, with non zero steering angle, possible equation is

$$
t_{L C}=\frac{-v \sin \left(\psi+\delta_{f_{0}}\right)+\sqrt{2 v^{2}\left(\frac{1}{R_{v_{d}}-\frac{a}{2}}-\frac{1}{R_{r}}\right) y_{l l}\left(\psi+\delta_{f_{0}}\right)+}}{v^{2}\left(\frac{1}{R_{v_{d}}-\frac{a}{2}}-\frac{1}{R_{r}}\right)}
$$

This equation is similar to equation (26), in which $v \sin \left(\psi+\delta_{f_{0}}\right)$ is the lateral speed, and $v^{2}\left(\frac{1}{R_{v_{d}}-\frac{a}{2}}-\frac{1}{R_{r}}\right)$ is the lateral acceleration relative to the road.

\section{B. Lateral speed and curvature estimation}

The bicycle model presents a state vector $x$ with 4 state variables. The state variables are the lateral speed, the lateral displacement, the relative yaw angle and the yaw rate. Only the three last variables are available for measurement. In addition, the road curvature affects the system as an unknown input [1].

$$
\left\{\begin{array}{l}
\dot{x}=A x+B \delta_{f}+E \rho_{r} \\
y=C x
\end{array}\right.
$$

An observer can be designed in order to achieve both state and unknown input estimation. In [8] a second-order 
polynomial model of the road is assumed in the vehicle coordinates. A discrete time Kalman filter observer is then synthesized for both lateral velocity estimation and polynomial model coefficients estimation. Another Kalman filter which considers road bank angle but ignores the road geometry in terms of curvature variation is also presented in [20]. Here it is assumed that the road curvature is almost constant or affine varying and we choose a proportional two-Integral (P2I) observer which is able to estimate the curvature and its derivative. The road curvature is considered as an unknown input $\rho_{r}$ [7].The P2I observer has the following form

$$
\left\{\begin{array}{l}
\dot{\hat{x}}=A \hat{x}+B \delta_{f}+L_{p}(y-\hat{y})+E \hat{\rho}_{r_{2}} \\
\dot{\hat{\rho}}_{r_{2}}=\hat{\rho}_{r_{1}}+L_{i_{2}}(y-\hat{y}) \\
\dot{\hat{\rho}}_{r_{1}}=L_{i_{1}}(y-\hat{y})
\end{array}\right.
$$

The second equation describes the integral loop gain added to the proportional one in the first equation. The matrix gains $L_{p}, L_{i 1}$ and $L_{i 2}$ are determined in such a way to enable asymptotic convergence to zero of the state estimation error, the unknown input estimation errors and the estimation error of the derivative of the unknown input, respectively defined by $e=x-\hat{x}, e_{\rho_{2}}=\rho_{r}-\hat{\rho}_{r_{2}}$ and $e_{\rho_{1}}=\dot{\rho}_{r}-\hat{\rho}_{r_{1}}$. This is achieved by making Hurwitz the matrix

$$
\left[\begin{array}{ccc}
A & E & 0 \\
0 & 0 & 1 \\
0 & 0 & 0
\end{array}\right]-\left[\begin{array}{c}
L_{p} \\
L_{i_{2}} \\
L_{i_{1}}
\end{array}\right]\left[\begin{array}{lll}
C & 0 & 0
\end{array}\right]
$$

Thus any eigenvalue assignment method can be applied to obtain the matrix gain $\left[\begin{array}{ccc}L_{p} & L_{i_{2}} & L_{i_{1}}\end{array}\right]$.

\section{Prediction of vehicle future positions}

For vehicle future positions dynamic prediction, the model (29) has to be transformed into a discrete-time model using the Tustin method. Prediction is performed during the time range in the frame attached to the road. This frame is obtained by the orthogonal projection of the vehicle CG on the lane centerline. This discrete model is of the form $x_{k+1}=\Phi x_{k}+\Gamma v_{k}$, where $x_{k}=\left[y_{G}, \dot{y}_{G}, \psi, \dot{\psi}\right]^{T}$ and $v_{k}=\left[\delta_{f}, \rho\right]^{T}$. This model is initialized using the observer estimation values for both the state and the road curvature [8]. This prediction is generally high computational and time consuming, it is only necessary in the cases where vehicle dynamics are far from steady state values and positioning values are critical. Prediction is performed until one of the vehicle front wheels reaches the lane edge or the fixed limit of time prediction has been completed [3], [2].

In the following, the established trigonometric formula are investigated in order to highlight effects of variables appearing in the different formula on the achieved TLC. Particularly, limits on acceptable relative yaw angle and lateral acceleration for minimum TLC values are outlined.

\section{Simulation ANALYSis}

\section{A. Analysis with zero steering angle on straight road section}

It is generally assumed that driver accuracy during lane keeping maneuvers is about $20 \mathrm{~cm}$ around the lane centreline at the vehicle center of gravity. On the other hand lane departures occur with a relative yaw angle under $6 \mathrm{deg}$. Let us define $d_{m}= \pm 20 \mathrm{~cm}$. Suppose that $\psi$ is positive, the lane departure will occur on the left side, and the point of interest is front left tire contact with the road. Vehicle geometry influences the lateral displacement of this point. This displacement is half the wheel-base at zero relative yaw angle and it increases about $17 \mathrm{~cm}$ when relative yaw angle is $10 \mathrm{deg}$. The point is located at a distance $\left(d_{m}+l_{f} \sin \psi+\frac{a}{2} \cos \psi\right)$ from the lane centreline, with $l_{f}=1 \mathrm{~m}$ and $a=1.4 \mathrm{~m}$. In order to maintain a time to line crossing of $t_{\min }=2 \mathrm{sec}$ for a given speed $v$, from equation (3), the relative yaw angle has to be under $\psi_{\max }$ given by

$$
\psi_{\max }=-\phi+\sin ^{-1}\left(\frac{\frac{L}{2}-d_{m}}{\sqrt{\left(v t_{\min }+l_{f}\right)^{2}+\frac{a^{2}}{4}}}\right)
$$

where the angle $\phi$ is such that $\sin \phi=\frac{\frac{a}{2}}{\sqrt{\left(v t_{\min }+l_{f}\right)^{2}+\frac{a^{2}}{4}}}$.

The relative yaw angle has to be under $2 \mathrm{deg}$ for speeds greater than $45 \mathrm{Km} / \mathrm{h}$, and only $1 \mathrm{deg}$ is acceptable at $90 \mathrm{Km} / \mathrm{h}$.

The decrease of time to line crossing according to relative yaw angle is now examined for a fixed speed $v=90 \mathrm{Km} / \mathrm{h}$ (figure 6). The solid line corresponds to vehicle located on the lane centerline while dashed line corresponds to vehicle center of gravity at $20 \mathrm{~cm}$ from centerline to the left. When the vehicle is on the centerline, a TLC of $2.4 \mathrm{sec}$ is obtained when relative yaw angle is $1 \mathrm{deg}$.

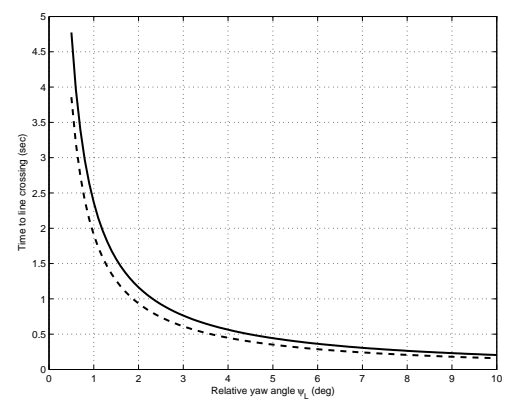

Fig. 6. TLC versus relative yaw angle for a fixed speed of $90 \mathrm{Km} / \mathrm{h}$ on straight road section and zero steering angle.

One can notice that the TLC decreases quickly and is less than $1 \mathrm{sec}$ beyond $2 \mathrm{deg}$ of relative yaw angle. Furthermore, effect of lateral displacement is more important for small values of $\psi_{L}$. For fixed speed and relative yaw angle, TLC is proportional to $y_{G l}$. As an example, a lateral displacement of $0.5 \mathrm{~m}$ leads to a TLC of only $2.25 \mathrm{sec}$ for $v=90 \mathrm{Km} / \mathrm{h}$ and $\psi_{L}=1 \mathrm{deg}$.

\section{B. Analysis with non-zero steering angle on straight road section}

From the kinematic model, the steady state value of the lateral acceleration is related to longitudinal speed and steering angle by

$$
\gamma_{l}=\frac{v^{2}}{l_{f}+l_{r}} \tan \delta_{f}
$$




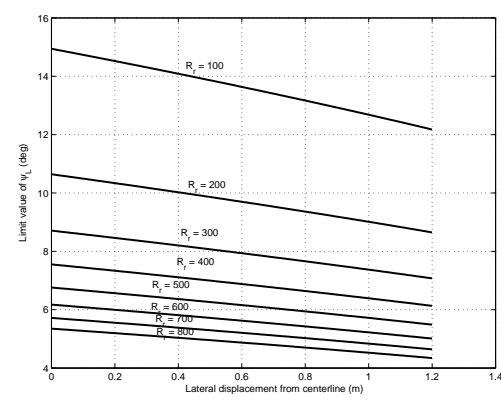

(a)

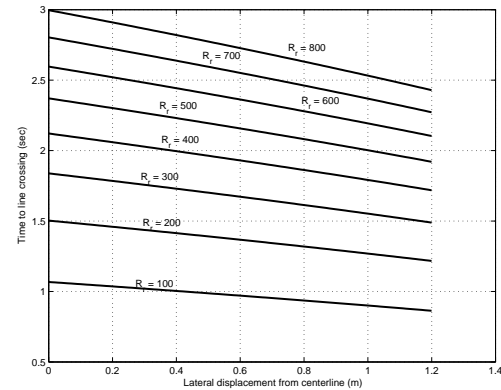

(b)

Fig. 7. (a)- Limit values of $\psi_{L}$ as a function of the lateral displacement for different road radius of curvature. (b)- TLC values obtained with the maximum value of $\psi_{L}$ as a function of the lateral displacement for different road radius of curvature.

where $l_{r}=1.4 \mathrm{~m}$.

In the following, the steering angle is chosen according to speed value such that $\gamma_{l}=3 \mathrm{~m} / \mathrm{s}^{2}$. The radius of curvature of vehicle path is computed from the stationary equality $\left(R_{v_{l}}^{-1}=\frac{\gamma_{l}}{v^{2}}\right)$.

We first assume that the relative yaw angle is zero. In this case, TLC is obtained from $t_{L C}=\frac{R_{v_{l}}}{v} \cos ^{-1}\left(\frac{R_{v_{l}}-y_{l l}}{R_{v_{l}}}\right)$. Results obtained when varying the speed show that TLC is almost constant if the lateral acceleration is maintained constant. This suggests that lateral acceleration can be locally used as an indicator of the TLC. On the other hand, one can also deduce from the previous equation which is the maximal lateral acceleration achieving a TLC of $2 \mathrm{sec}$. Assuming a $20 \mathrm{~cm}$ lateral displacement of $\mathrm{CG}$, the obtained lateral acceleration is $\gamma_{l}=4.2 \mathrm{~m} / \mathrm{s}^{2}$.

Finally, simulations show that a non zero relative yaw angle leads to a linear decreasing of TLC as the speed increases. The slope is almost proportional to the relative yaw angle.

\section{Analysis on curved road sections}

It has been shown previously (table III), that lane departure on the left side is avoided provided that $\psi_{L}<\psi_{t l}=$ $\cos ^{-1}\left(\frac{R_{r}}{R_{r}+y_{l l}}\right)$. Figure 7 -a gives the limit values for $\psi_{L}$ as a function of $y_{l l}$ for different values of curve radius $R_{r}$.

The corresponding TLC limit is shown in figure 7-b for a speed of $90 \mathrm{Km} / \mathrm{h}$. It is obtained on curved road section assuming straight vehicle trajectory with initial relative yaw angle $\psi_{t l}$. One can notice that for highway curvatures, the relative yaw angle has to be under $5 \mathrm{deg}$ in order to ensure a TLC of more than $2 \mathrm{sec}$.

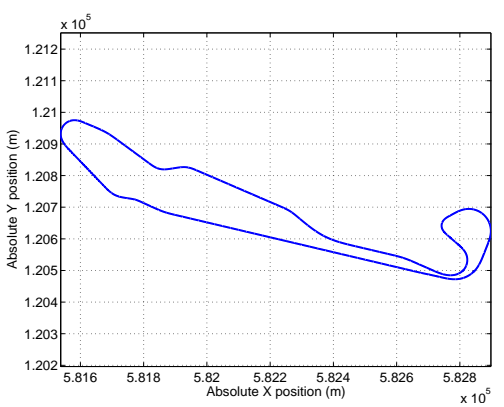

(a)

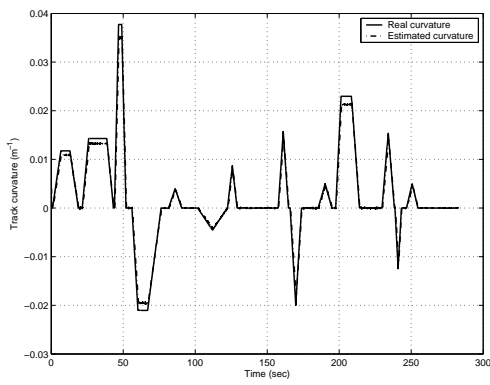

(b)

Fig. 8. (a)- Digitalized map of the LIVIC test track. (b)- Real and estimated track curvature.

\section{Simulation based evaluation on test track profile}

In 1999, INRETS established a test track in Satory, 20Km western Paris. The site is $3.5 \mathrm{Km}$ long with various road profiles including straight lane, tight bend and squabble (figure 8a). Lanes markers absolute positions were digitalized each $5 \mathrm{~cm}$ using differential GPS (DGPS). In addition, an experimental vehicle is equipped with video cameras on each side which can detect lane markers at vehicle CG with high accuracy [6].

Figure 8-b shows in solid line the curvature of the track. The P2I observer developed above is able to estimate this curvature using only video sensor (lateral displacement and relative yaw angle measurement) and the gyro as shown in figure 8-b. Figure 9-a give a zoom of the estimation between time $110 \mathrm{sec}$ and $190 \mathrm{sec}$. For better precision with the lateral positioning, the lateral displacement is measured with 2 vertical cameras at vehicle center of gravity. Figure 9-b shows that the observer is able to estimate the curvature derivative. The results obtained with the observer permit to trust that the DLC based computation of the TLC can be implemented in near future without use of DGPS and digitalized maps if the lane edge are of sufficient quality in order to be detected by video sensors.

Figure 10-a characterizes the test track in terms of distance to line crossing, when it is assumed that the vehicle is positioned on the centerline with zero relative yaw angle. This distance is greater than $300 \mathrm{~m}$ and reaches $900 \mathrm{~m}$ on the straight sections of the track (see figure 8-b for the corresponding curvature). However, DLC is several times under $100 \mathrm{~m}$ which means that small TLC values may be expected for high speeds or when lateral positioning or relative yaw angle errors are taken into account. 


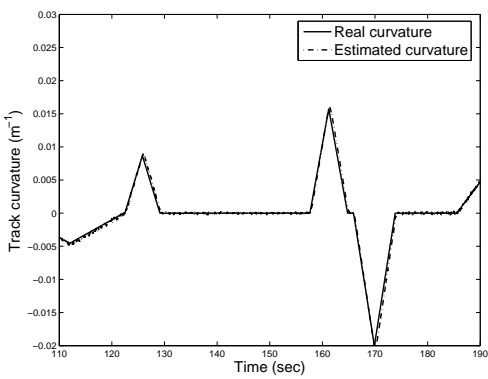

(a)

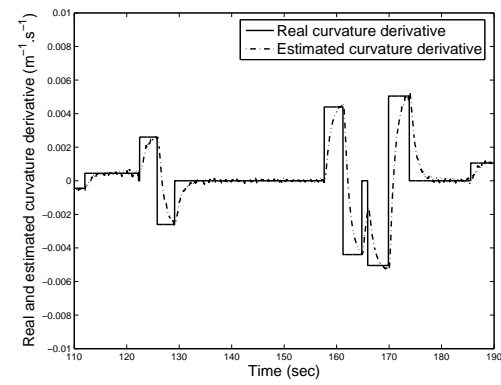

(b)

Fig. 9. (a)- Zoom on the real and estimated track curvature. (b)- Real and estimated curvature derivative.

Speed is intentionally limited to $144 \mathrm{Km} / \mathrm{h}(40 \mathrm{~m} / \mathrm{s})$ on straight sections, while on curved section the forward speed is chosen in order to obtain a constant lateral acceleration of $0.3 \mathrm{~g}$. The obtained speed profile is used in various forms in all TLC computations. Evaluation will be performed on the section from arc length $1500 \mathrm{~m}$ until the end of the track (figure 10-b).

First, the vehicle is considered at the center of the lane with zero relative yaw angle. TLC values are shown in figure 11-a.

The steering angle is now chosen at a nominal value according to equation (7), in order to obtain a vehicle radius path equal to track curvature at vehicle location. TLC results are shown in figure 11-b. TLC is particularly enhanced on curved sections.

Vehicle relative lateral displacement is introduced of the form of a sinusoidal wave with $20 \mathrm{~cm}$ magnitude. Results are provided in figure 11-c. The effect on TLC is only visible in the region between the two vertical bars.

A supplementary Gaussian random relative yaw angle error is also introduced. Variance is chosen at $1 \mathrm{deg}$ and the obtained TLC is shown in figure 11-d. One can notice high degradation of TLC values especially on straight section.

\section{EXPERIMENTAL VALIDATION}

In the previous parts, we have discussed about the theoretical computation of approximations of TLC. In practice, two classes of hypothesis could be used in the computation of the TLC.

The first class is about road consideration. It depends on the road sensor available in the vehicle. With only a short range sensor, the road is considered as a straight line as we can only measure the lateral displacement and the road relative yaw angle. Long range sensors give us the knowledge of the

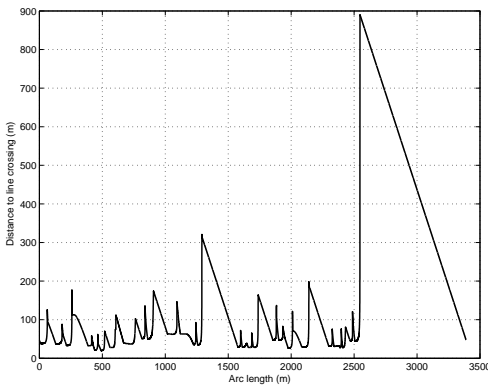

(a)

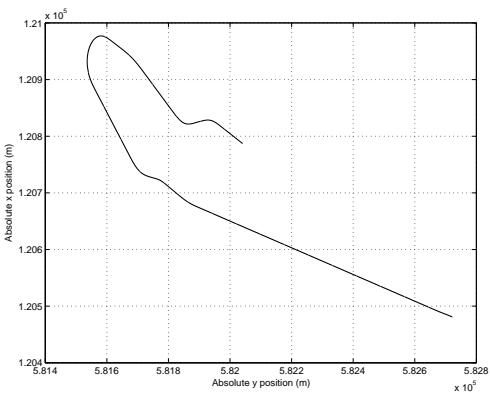

(b)

Fig. 10. (a)- Distance to line crossing along the track, when it is assumed that the vehicle is positioned on the centerline with zero relative yaw angle. (b)- Section of track on which TLC is computed.

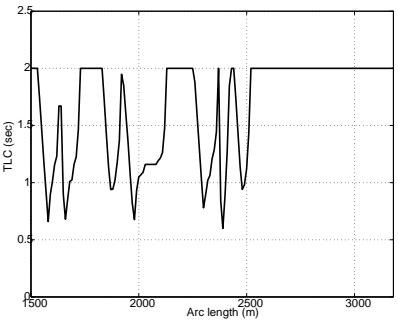

(a)

(c)

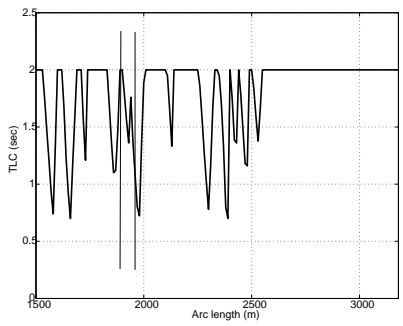

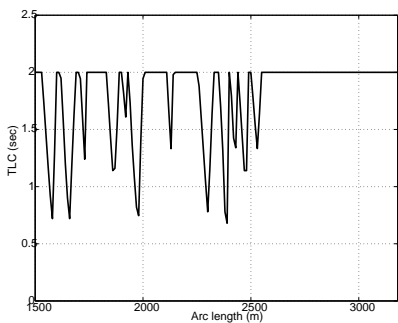

(b)

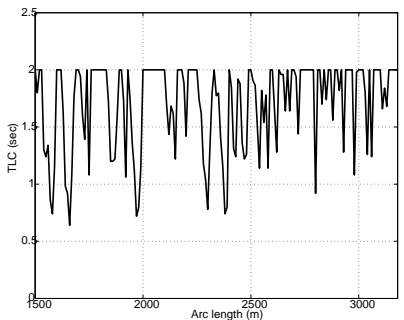

(d)
Fig. 11. (a)- TLC values for vehicle on centerline with zero relative yaw angle and zero steering angle. The lateral acceleration is $0.3 \mathrm{~g}$. (b)- TLC values for vehicle on centerline with zero relative yaw angle and nominal steering angle. The lateral acceleration is $0.3 \mathrm{~g}$. (c)-Vehicle within $20 \mathrm{~cm}$ from the centerline of the lane, zero relative yaw angle and nominal steering angle. The lateral acceleration is $0.3 \mathrm{~g}$. (d)-Vehicle within $20 \mathrm{~cm}$ from the centerline, Gaussian noise relative yaw angle and nominal steering angle. The lateral acceleration is $0.3 \mathrm{~g}$. 


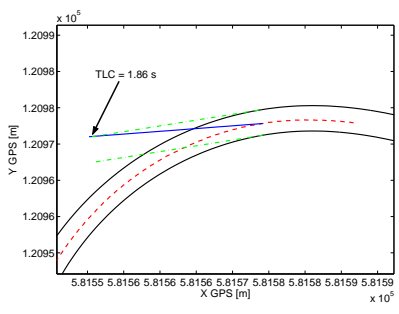

(a)

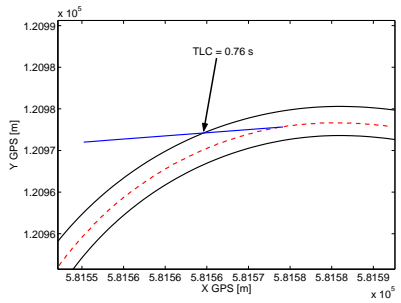

(c)

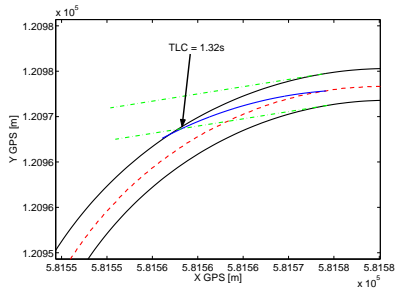

(b)

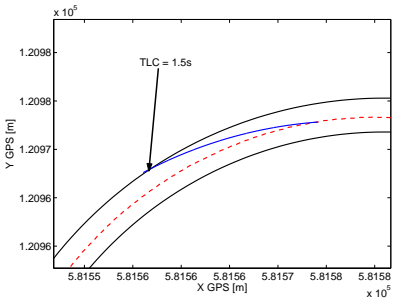

(d)
Fig. 12. Different TLC approaches on a curve. (a)- Straight road and vehicle trajectory approximations. (b)- Straight road approximation and curved vehicle trajectory. (c)- Real road profile and straight vehicle trajectory approximation. (d)- Real road profile and curved vehicle trajectory.

road position, and we can fully take into account the road description.

The second class is relative to the vehicle knowledge. Without any sensor, we can make no assumption on the vehicle future path, so we consider it as a straight line. With sensors, such as yaw rate sensor, speedometer and steering angle, it is possible to predict the vehicle path for TLC computation. As mentioned before, advanced dynamic model prediction can be performed by observer based state and curvature estimation.

Figure 12 shows these different modes of TLC computations:

- In (a), the TLC is computed considering straight road approximation and straight vehicle trajectory (denoted $\mathrm{LD} / \mathrm{LD}$ in future plots),

- In (b), the TLC is computed considering straight road approximation and curved vehicle trajectory (denoted $\mathrm{LD} / \mathrm{Ce})$

- In (c), the TLC is computed considering real road profile and straight vehicle trajectory (denoted RR/LD),

- In (d), the TLC is computed considering real road profile and curved vehicle trajectory (denoted RR/Ce),

This set of figures shows the applied TLC computation on a curve. Consideration of the road in this case is very significant and leads to large variations in the TLC values.

The following parts will develop experimental validation of TLC computation. The main problem is to know which computation is the most efficient according to driving behavior.

This part is devoted to the definition of the experimental test to validate and compare the different TLC. We present in the first subsection the sensors used for the TLC computation. We explain then the basic scenarios used for the validation of the TLC.

\section{A. Sensors and Scenarios}

In order to ensure a high accuracy on the vehicle position and road description, we use a digital map and a centimeter
TABLE VI

GPS ACCURACY VALIDATION

\begin{tabular}{|c|c|c|c|c|}
\hline \multicolumn{2}{|c|}{ distance $(\mathrm{cm})$} & \multicolumn{2}{|c|}{ lateral displacement $(\mathrm{cm})$} & \multirow{2}{*}{$\begin{array}{c}\text { absolute } \\
\text { difference }(\mathrm{cm})\end{array}$} \\
\hline left & right & measured & rounded GPS & \\
\hline 83 & 63 & -10 & -9 & 1 \\
\hline 70 & 76 & 3 & 3 & 0 \\
\hline 78 & 68 & -5 & -5 & 0 \\
\hline 79 & 67 & -6 & -6 & 0 \\
\hline 50 & 96 & 23 & 24 & 1 \\
\hline 64 & 82 & 9 & 9 & 0 \\
\hline 67 & 79 & 6 & 6 & 0 \\
\hline 89 & 57 & -16 & -16 & 0 \\
\hline 81 & 65 & -8 & -9 & 1 \\
\hline 64 & 82 & 9 & 10 & 1 \\
\hline
\end{tabular}

RTK-GPS. These components are described below:

1) Digital Map: The maps, used for the experimental part, contain the position of the road edges and the center of the road. Positions are given with a longitudinal step of fifty centimeter and the accuracy is about one centimeter.

2) GPS: In order to localize the vehicle on the map, a RTK GPS is used. The acquisition sampling frequency of the vehicle position is $20 \mathrm{~Hz}$ and the accuracy is one centimeter due to the short distance to the base station. Accuracy of the vehicle localization on the map using the RTK GPS has been validated. This GPS was installed in a vehicle driving at about $50 \mathrm{~km} / \mathrm{h}$. The RTK GPS and the map have been used to compute lateral displacement of the vehicle with respect to the center of the road. In order to validate this measure, the lateral displacement was also computed using an external measure. This one was obtained by an experimental process that consisted of covering a part of the lane with sand and observing the marks that the vehicle left on this sand. Table VI summarizes ten measures of lateral displacement using both methods. All measures are given in centimeters, the difference between the two methods being rather small, with a mean of 0.4 centimeters. The centimeter accuracy of the GPS and map system is then validated.

The GPS centimeter mode is available on a large part of the test track, presented previously. Data used to assess the centimetric precision on the test track have been recorded during a one week test. Measures have been taken from 9:00 in the morning to 19:00 under various conditions, with the vehicle speed reaching $20 \mathrm{~m} / \mathrm{s}$. More than 200 turns have been done. This long period ensures us to collect data from various GPS satellite constellations. Data used in the next parts are taken on sections with centimetric positioning.

3) Scenario: The tested scenarios represent common driving situations, with respect to lane departure problems. To validate the TLC approach, in the first scenario, the driver is asked to drive normally, following the center of the road. In this scenario, the TLC is expected to have normal values, with small variations. Two driver styles were chosen for this scenario: low and high speeds.

In the second scenario, we wanted to test the reaction of the TLC, with respect to a driver correction during a slowly lane departure situation. So, the vehicle goes slowly near the road mark, and the driver has to correct this situation.

Finally, in the last scenario, the driver must cross the lane. 


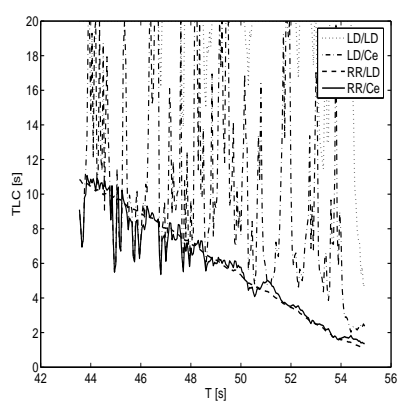

(a)

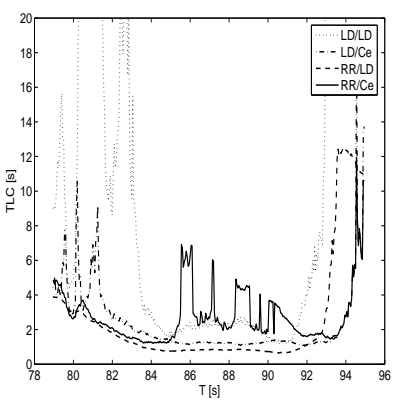

(b)
Fig. 13. TLC variation during a normal driving scenario. (a)- Drive on a straight line. (b)- Drive on a curve.

\section{B. Results}

1) Normal driving condition: Driver is asked to follow the center of the road, with an average speed of $50 \mathrm{Km} / \mathrm{h}$.

Two relevant situations are plotted in figure 13. A long straight line, ending with a curve is shown on figure 13-a. Figure 13-b represents the values of the TLC on a curve. A first remark is that the values of the TLC are not small, as the driver, in this test, does not take risk. On these two plots, the TLC computed using the approximation of straight road may lead to large values and large variations of TLC. Since in this case, the driver follows the center of the road, his relative yaw angle is small. As these approximations of the TLC are strongly dependent on this angle, a small variation of this variable has a large impact. On the other hand, information given using real road profile is reliable. On the straight line, the vehicle speed is approximatively constant. So, his distance to line crossing decreases with a constant speed. In the time versus time representation of figure 13.(a), the slope of the TLC for these two approximations is near 1.

2) Slow road departure and driver correction: In this scenario, the driver goes near the lane marks but never crosses them. Figures 14 and 15 show the vehicle trajectory and the related TLC. In both figures, the driver has performed a correction after a slow lane departure, which is the greater cause of lane departures.

With respect to the figure 14, all TLC computations show good response to this problem. Values of different computations are similar: in this case, both road profile and vehicle trajectory are about a straight line before the driver correction. Moreover, the DLC is small. So, the different approximations on the vehicle trajectory and on the road profile lead to similar results. By using the curvature of the trajectory, the reaction in the TLC increase as a consequence of the driver correction is faster of $0.3 \mathrm{sec}$ than with the straight trajectory approximation.

In figure 15, excepting the TLC computed with straight road and straight trajectory approximation, all computations show a risky situation, with small TLC values (below $2 \mathrm{sec}$ and even $1 \mathrm{sec})$. If we do not take into account the curvature of the trajectory in the TLC computation, we can see that the TLC does not reflect quite well the vehicle movement approaching the road marks. Moreover, the computation using real road profile and straight driver trajectory, shows false variations: just before $T=56 \mathrm{sec}$, the driver corrects his trajectory and moves away from the road mark, but the TLC value does not stop decreasing.

3) Road departure: In this last scenario, the driver runs off the road. This road departure case simulates the loss of control, for instance on icy road.

Figure 16 shows the vehicle trajectory and the TLC values. All TLC computations drop to zero when the vehicle crosses the road mark. Since the vehicle speed is constant, the slope of the TLC is near 1 . The computation, using the vehicle trajectory curvature and the real road, shows a step of one second in TLC, two seconds before the lane departure. This step results of a driver correction. The TLC computation using both straight line approximations, show bad results as the TLC values, $2 \mathrm{sec}$ before the lane crossing are very high and not representative of the emergency of the situation.

Developing a lane departure unit is a big challenge for accident and death reduction. The lane departure prevention unit has to provide an information or an alarm to the driver that takes into account several constraints

- Excessive speed even on straight road section

- Vehicle positioning: excessive lateral displacement and excessive relative yaw angle

- Excessive lateral acceleration

- Small TLC values.

Decision making strategies for lane departure systems have been investigated by several authors [19],[22]. A lane departure warning unit which takes into account the fourth items is under validation [23].

\section{CONCLUSION}

In this paper, TLC computation is presented on the basis of geometric kinematic formula and dynamic trajectory prediction. It is pointed out that all necessary data can be easily measured by a video sensor or DGPS, or estimated by using a model based observer. Parameter effects analysis show that the choice of one computation approximation for TLC is conditioned by the driving situation type and road characteristics. High accuracy measure of vehicle positioning is also needed particularly in terms of relative yaw angle. Experimental validation has not clearly highlighted one TLC computation as the best, but has allowed us to draw aside the straight road approximation. In fact, this approximation brings a too large variation in the TLC values and gives us TLC which are not representative of the situation. This approximation has been considered to take into account sensors capacity: as a matter of fact, it is easier to obtain the road profile at the location of the vehicle rather than in front of the vehicle. Using the real road profile, the approximation on vehicle trajectory leads to different results. With a straight vehicle trajectory, the TLC plot is smooth and presents good characteristics, but, compared to the curved trajectory, the reaction to driver correction is delayed. This study represents a first step in the use of TLC as a driver risk indicator. We have carried it out using a RTK GPS and an accurate map. In order to be largely used, the TLC implementation must require more common sensors, such as camera-based lane detection as well as cooperative systems or the use of observer based methods. 


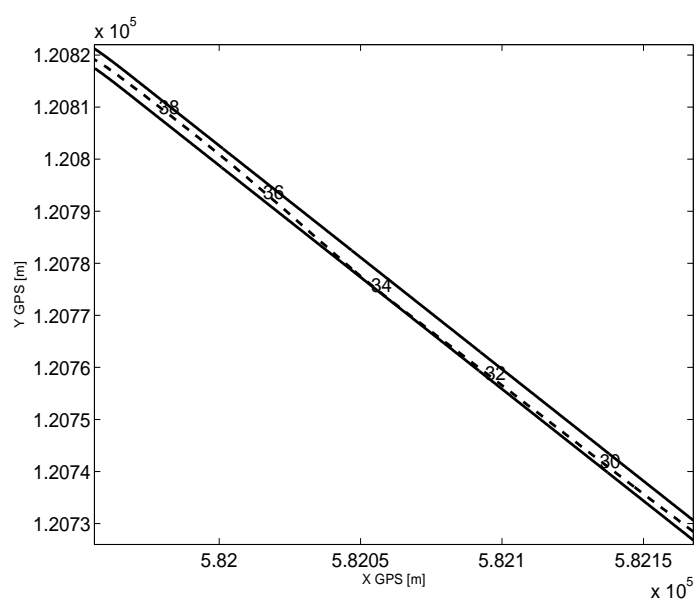

(a)

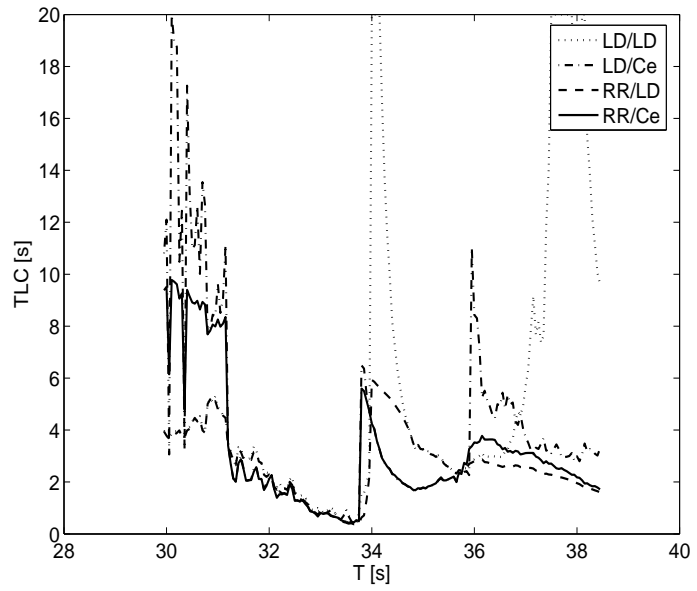

(b)

Fig. 14. Slow road departure and driver correction scenario. (a)- Vehicle trajectory. (b)- Related TLC variation.

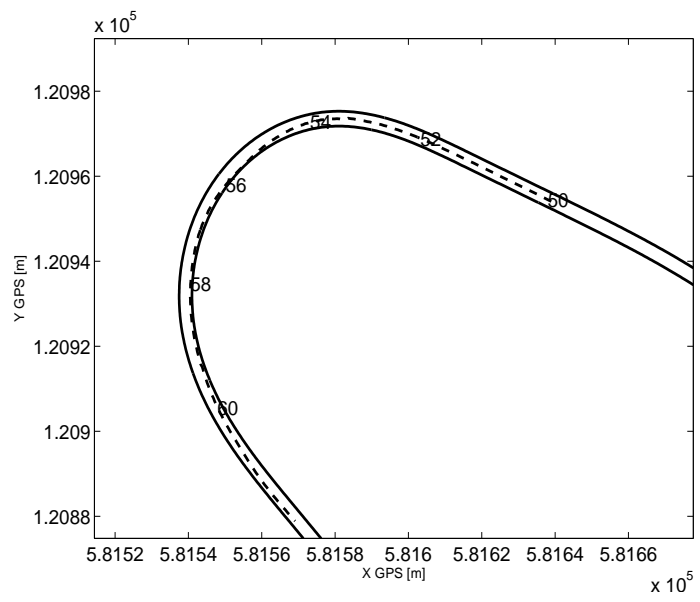

(a)

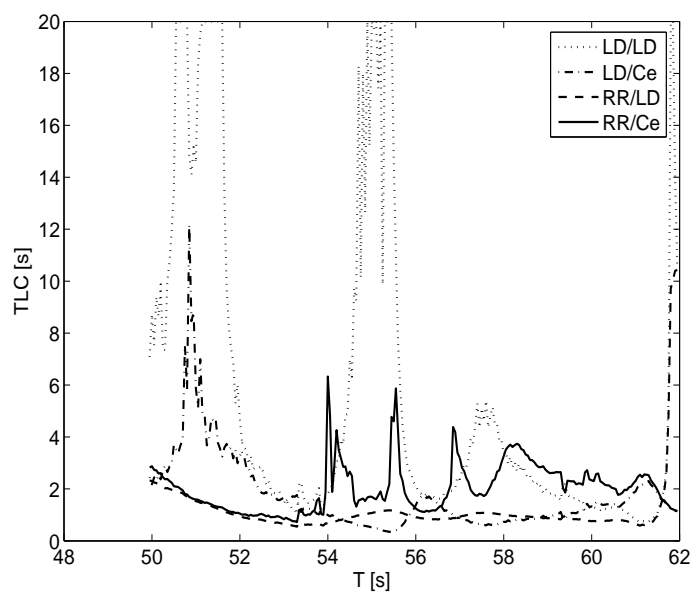

(b)

Fig. 15. Slow road departure and driver correction scenario. (a)- Vehicle trajectory. (b)- Related TLC variation.

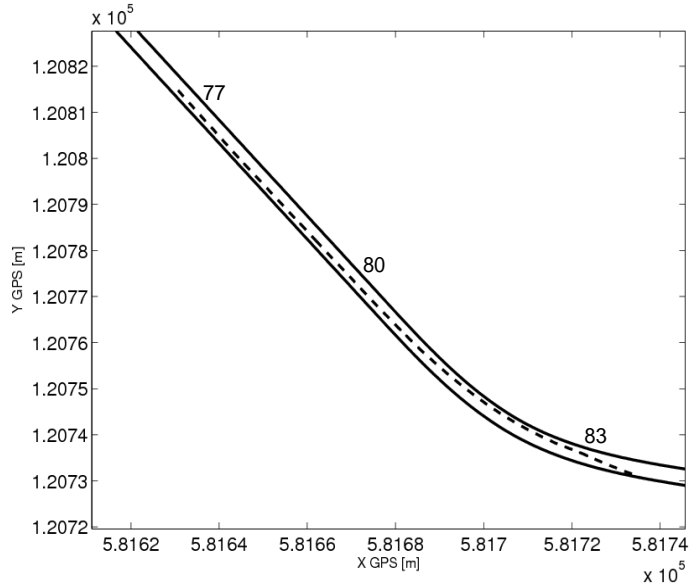

(a)

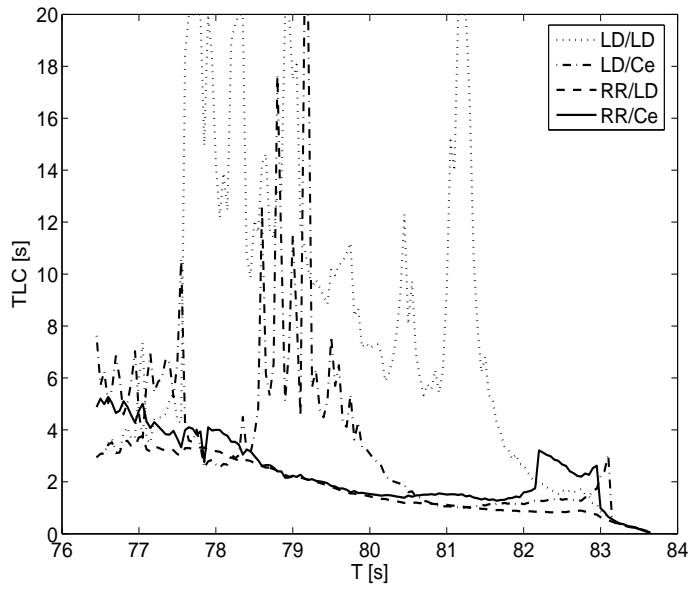

(b)

Fig. 16. Road departure driving scenario. (a)- Vehicle trajectory. (b)- Related TLC variation. 


\section{REFERENCES}

[1] J. Ackermann, et.al.,"Linear and nonlinear controller design for robust automatic steering," IEEE Transactions on Control Systems Technology, Special Issue on Automotive Control, vol. 3, pp. 132-143, 1995.

[2] K. T. Feng , "Look-ahead Human machine Interface for assistance of manual vehicle steering," American Control Conference, 1999.

[3] K.T. Feng, H.S. Tan and M. Tomizuka, "Futur Predictor for Vehicle Steering Guidance-Sensitivity Analysis and Experimental Results," Conference on Decision and Control, 1999.

[4] J.J. Gibson, "The perception of the visual world," Houghton, Mifflin, 1950.

[5] H. Godthelp, P. Milgram, and G. J. Blaauw, "The development of a time Related Measure to describe driving strategy," Human Factors, 263, 257-268, 1984.

[6] S.S. Ieng and D. Gruyer, "Merging lateral cameras information with proprioceptive sensors in vehicle location gives centrimetric precision," ESV 2003, Nagoya, Japan, 2003.

[7] D. Koenig and S. Mammar, "Design of Proportional-Integral Observer for Unknown Input Descriptor Systems", IEEE Transactions on Automatic Control, vol 47, No 12, 2057 - 2062, 2002.

[8] C.-F. Lin , A.G. Ulsoy, and D.J. LeBlanc, "Vehicle Dynamics and External Disturbance Estimation for Vehicle Path Prediction," IEEE Transactions on Control System Technology, vol 8, 3, 508-518, 2000.

[9] C.F. Lin, and A. G. Ulsoy, "Time to lane crossing calculation and Characterization of its associated uncertainty," ITS Journal, 3-2, 85-98, 1996.

[10] D.R. Mestre, "Dynamic evaluation of the useful field of view in driving," Proceedings of Driving Assessment, 234-239, 2001..

[11] I. Milleville-Pennel, J.M. Hoc and F. Mars, " Useful sensory information for trajectory control while taking a bend", Internal Report IRCCyN UMR CNRS - Equipe PsyCoTec, Nantes, France, 2004

[12] B.J. Rogers, and R.S. Allison, "When do we use optical flow and when do we use perceived direction to control locomotion? ", Perception, 28, S2, 1999.

[13] W. Van Winsum, K. A. Brookhuis and D. de Waard, A comparison of different ways to approximate time-to-line crossing (TLC) during car driving, Accident Analysis and Prevention, 32, 47-56, 2000.

[14] W.V. Van Winsum and H. Godthelp, "Speed choice and steering behavior in curve driving," Human Factors, 38, 434-441, 1996.

[15] W.V. Van Winsum, D. de Waard, and K.A. Brookhuis, "Lane change manoeuvres and safety margins," Transportation Research Part F, 2, 139-149, 1999.

[16] P. H. Batavia, Driver-Adaptive Lane Departure Warning Systems, Carnegie Mellon University, Ph D., 1999.

[17] P. H. Batavia and D. Pomerleau and C. Thorpe, Predicting Lane Position for Roadway Departure Prevention, Proceedings of the IEEE Intelligent Vehicles Symposium, 1998.

[18] M. Chen and T. Jochem and D. Pomerleau, AURORA: A Vision-Based Roadway Departure Warning System, IEEE Conference on Intelligent Robots and Systems, Human Robot Interaction and Cooperative Robots, 1998.

[19] W. Kwon and J. W. Lee and D. Shin and K. Rob and D. Y. Kim and S. Lee, Experiments on Decision Making Strategies for a Lane Departure Warning System, IEEE International Conference on Robotics and Automation, 1999.

[20] N. Mudaliar, D. LeBlanc and H. Peng, Linear Estimator for Road Departure Warning Systems, American Control Conference, 2004, pp. 2104-2109, 2004

[21] D. B. Pape, V. K. Narendran, M. J. Koenig, J. A. Hadden, and J. H. Everson, Dynamic Vehicle Simulation to Evaluate Countermeasure Systems for Run-Off-Road Crashes, SAE International Congress 81Exposition, Detroit, Michigan, 1996.

[22] S. Lee, and W. Kown, Performance Evaluation of Decision Making Strategies for an Embedded Lane Departure Warning System, Journal of Robotic Systems, v 19, n 10, pp. 499-509, 2002.

[23] C. Sentouh, S. Glaser, S. Mammar, Lane Departure Warning Unit, Design and Evaluation, Submitted for intteligent Vehicle Symposium, IV'2006, 2006.

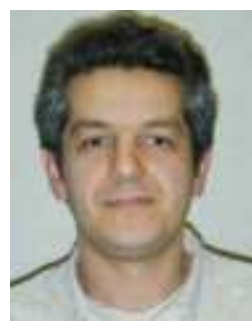

S. Mammar received the Dipl.-Ing degree from École Supérieure d'Électricité, Gif-sur-Yvette, France in 1992, the Ph.D degree in automatic control from Université Paris XI-Supelec, Orsay, France in 1992 and the Habilitation to Direct Research (HDR) from Université d'Evry, Evry, France in 2001.

From 1992 to 1994, he held a research position at INRETS, the French National Institute on Transportation Research and Safety, France, where he worked on traffic network control. From 1994 to 2002, he was an Assistant Professor at Université d'Évry, Évry, France. He is currently a professor and head of the Department of Electrical Engineering at Université d'Évry, Évry, France. His actual research interests include robust control, vehicle longitudinal and lateral control for driving assistance and Intelligent Transportation Systems. He is currently involved in the integrated European Project PReVENT.

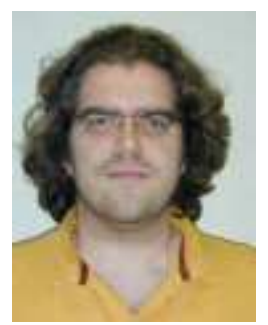

S. Glaser received the Dipl.-Ing. Degree from the École Nationale des Travaux Publics de l'État, Lyon, France in 2001 and his Master Degree in Vision from the University of Saint Étienne, France, the same year. He received the $\mathrm{Ph} \mathrm{D}$. degree in Automatic Control from the University of Évry, France, in 2004.

Since 2001, he is with the Vehicle-InfrastructureDriver Interactions Research Laboratory (LIVICLCPC/INRETS), in Versailles, France. His actual research interests include Intelligent Transportation Systems, Vehicle modelling and control with driver interaction. He is currently involved in the Integrated European Projects SafeSpot and CVIS.

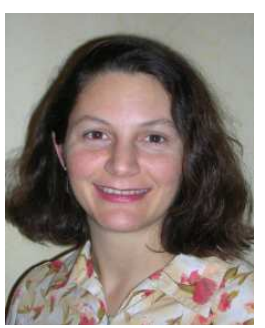

M. Netto received the B.Sc. degree in electronic engineering in 1995 and the M.Sc. degree in automatic control, in 1997, both from the Federal University of Rio de Janeiro, Brazil, and the Ph.D. degree in automatic control, in 2001, from the Laboratoire des Signaux et Systèmes (L2S) - Supélec - Université Paris Sud, France.

She is currently a researcher in the VehicleInfrastructure-Driver Interactions Research Laboratory (LIVIC-LCPC/INRETS), in Versailles, France. Her research interests include nonlinear and adaptive control, full and shared lateral control for driver assistance systems and intelligent transportation systems. She is currently involved in the Integrated European Project PReVENT. 